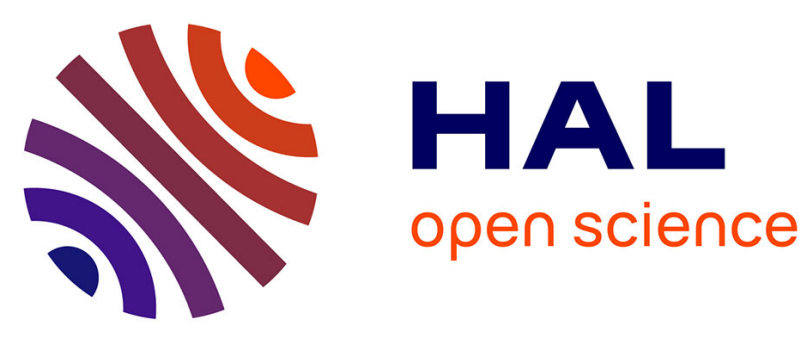

\title{
Fine-Grained Intercontact Characterization in Disruption-Tolerant Networks
}

Tiphaine Phe-Neau, Marcelo Dias de Amorim, Vania Conan

\section{To cite this version:}

Tiphaine Phe-Neau, Marcelo Dias de Amorim, Vania Conan. Fine-Grained Intercontact Characterization in Disruption-Tolerant Networks. IEEE Symposium on Computers and Communications, Jun 2011, Kerkyra, Greece. pp.271 - 276, 10.1109/ISCC.2011.5983789 . hal-00620831

\section{HAL Id: hal-00620831 \\ https://hal.science/hal-00620831}

Submitted on 9 Sep 2011

HAL is a multi-disciplinary open access archive for the deposit and dissemination of scientific research documents, whether they are published or not. The documents may come from teaching and research institutions in France or abroad, or from public or private research centers.
L'archive ouverte pluridisciplinaire HAL, est destinée au dépôt et à la diffusion de documents scientifiques de niveau recherche, publiés ou non, émanant des établissements d'enseignement et de recherche français ou étrangers, des laboratoires publics ou privés. 


\section{Fine-Grained Intercontact Characterization in Disruption-Tolerant Networks}

\author{
Tiphaine Phe-Neau \\ UPMC Sorbonne Universités \\ tiphaine.phe-neau@lip6.fr
}

\author{
Marcelo Dias de Amorim \\ CNRS and UPMC Sorbonne Universités \\ marcelo.amorim@lip6.fr
}

\author{
Vania Conan \\ Thales Communications \\ vania.conan@fr.thalesgroup.com
}

\begin{abstract}
So far, efforts attempting to characterize the spatiotemporal nature of disruption-tolerant networks (DTN) have relied on the dual notion of contacts and intercontacts. A contact happens when two nodes are within communication range of each other. An intercontact is simply defined as the dual of a contact, i.e., when two nodes are not in communication range of each other. We refer to this model as "binary". Although the binary characterization allows understanding the main interaction properties of the network, it is not sufficient to capture a plethora of situations beyond the binary hypothesis. In this paper, we investigate the structural properties of the network when nodes are not in contact but do have a contemporaneous path connecting them. We first introduce the notion of $n$ ary intercontact and, to defend its adoption, we quantify the proportion of nodes bearing this new intercontact notion in well-known datasets available to the community. Surprisingly, we observe that most pairs of nodes are nearby (within a few hops) for significant amounts of time when not directly in contact. Finally, we compare the impact of our proposal with the classic intercontact definition and give incentives toward using the $n$-ary characterization to leverage new communication opportunities.
\end{abstract}

\section{INTRODUCTION}

With the growing penetration of intelligent devices like smartphones, tablets, or portable game stations into people's everyday life, disruption-tolerant networking (DTN) emerged as a groundbreaking communication paradigm in the modern networking landscape [1]. To satisfy the communication needs among those devices in an opportunistic way, many new stimulating approaches have been proposed in the literature [2], [3], [4], [5]. A common substrate to these approaches is to rely on the complementary concepts of contacts and intercontacts. The contact notion has a factual definition, it is when two nodes are within direct communication range of each other. An intercontact is defined as the complementary of a contact, i.e., simply when two nodes are not in contact. As we will see in this paper, such a simplistic definition for intercontacts (henceforth mentioned as binary intercontacts) ends up being a melting pot for any attempt to benefit from the geographic proximity of users.

Fig. 1 represents a network snapshot illustrating our concerns. From A's point of view, it has two nodes in contact (nodes B and C). With the binary intercontact definition, all four remaining nodes are considered in "intercontact" mode. In such a situation, most DTN approaches infer the impossibility of exchanging messages via multi-hop paths and often calls for a "wait" period until it meets the destination or find someone

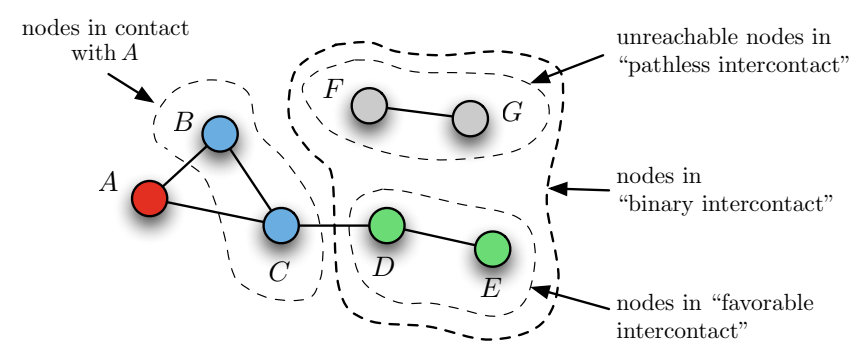

Fig. 1. Node A neighborhood "contact and intercontacts": A is in contact with his two direct neighbors, all four remaining nodes are in binary intercontact. However, we can reach the lower pair of nodes in intercontact via a contemporaneous path, they are in a favorable intercontact state.

else that knows the destination better (based on some other criterion). By denying the inherent ad hoc network part in DTN, we cannot pull the best of both worlds. In our example, we observe that ad hoc communication would succeed if $\mathrm{A}$ had data to transfer to either D or E. Contemporaneous paths may exist between nodes not in contact. We refer to this property as favorable intercontact. A natural question that arises is: should node A use multi-hop communications to send data to D and $\mathrm{E}$ or wait until it gets within direct communication range with them?

In this paper, we tackle the fundamental problem of knowing whether a pair of nodes shows sufficient sociability to spend a significant share of time in the vicinity of each other. To this end, we investigate several real-world datasets available to the research community and provide insights into fine details of intercontact periods. We formalize our proposal by defining the notion of $n$-ary intercontacts, where $n$ stands for the distance separating two nodes.

Our work has its foundations on several previous contributions of the literature. As outlined many times, user mobility patterns are not random [6]. People have a tendency to form communities and then display "favorable" intercontact properties. Gaito et al. based their study on workplaces as they also felt the force of social patterns in DTN [7]. There are induced relationships between human-carried devices. Other works, such as the ones of Whitbeck et al. [5] and Borrel et al. [8] propose to classify opportunistic networking into several categories depending on the expected group formation when nodes move around. Our work is complementary to the aforementioned ones as it makes a step further by analyzing 
node proximity in a pairwise way and by identifying "hidden intercontact possibilities" that are often underestimated by traditional forwarding solutions.

We make several interesting observations in our study. Firstly, we confirm that the binary contact-intercontact idea is too rough to efficiently capture potential communication opportunities while nodes are not in contact. Secondly, for a significant amount of pairs, we observe that nodes spend as much time at 1 and 2-hop distances. Thirdly, for some datasets, about half of the traditional intercontact time is in fact a connected. We do believe that our results will motivate protocol designers to first check close neighborhood before adopting a wait strategy.

In summary, the contributions of this paper are:

- New ternary vision for accurate DTN understanding through $n$-ary intercontact characterization.

- Empirical analysis to show that the binary contact assumption is not enough via an evaluation of binary vs. $n$-ary intercontact behaviors in existing datasets.

- Extended comparison between binary and pathless intercontact distributions.

\section{N-ARY INTERCONTACT: DEFINITION AND OBJECTIVES}

In this section, we provide the necessary background as well as an intuitive explanation of our DTN characterization.

\section{A. Pairwise visualization}

Considering a high level abstraction for intercontact erases punctual events happening between given nodes (for instance, people eating at the same place, at the same time, every week day). Yet, we could use them for efficient forwarding. To maintain traces of these events, we choose to perform pairwise analysis. Bearing in mind the inaccuracy of the binary intercontact definition, we thought of $n$-ary intercontact as a reflector of human sociostructure as well as ad hoc communication opportunities. We divide it into two intuitive notions: favorable and pathless intercontacts.

Definition 1. Favorable intercontact. An intercontact is considered as "favorable" with parameter $n$ when there is a contemporaneous shortest path of length $n \in[2 ; \infty[$ separating the two nodes under consideration.

Note that the parameter $n$ is a key ingredient in our study. As we will see later, this characterization provides richer information to help decide which forwarding strategy a protocol should use. Depending on the time spent at a certain distance, we can also derive other parameters such as the surrounding stability of a node. Favorable intercontact can also be seen as extended ad hoc communication opportunities.

Definition 2. Pathless intercontact. In opposition to favorable situations, "pathless" intercontact indicates the lack of endto-end paths between a pair of nodes, i.e., $n=\infty$.

This granularity reveals real situations in the DTN context and can influence a node's resolution to use asynchronous transmission scheme from delaying transmission to specific routing approaches.

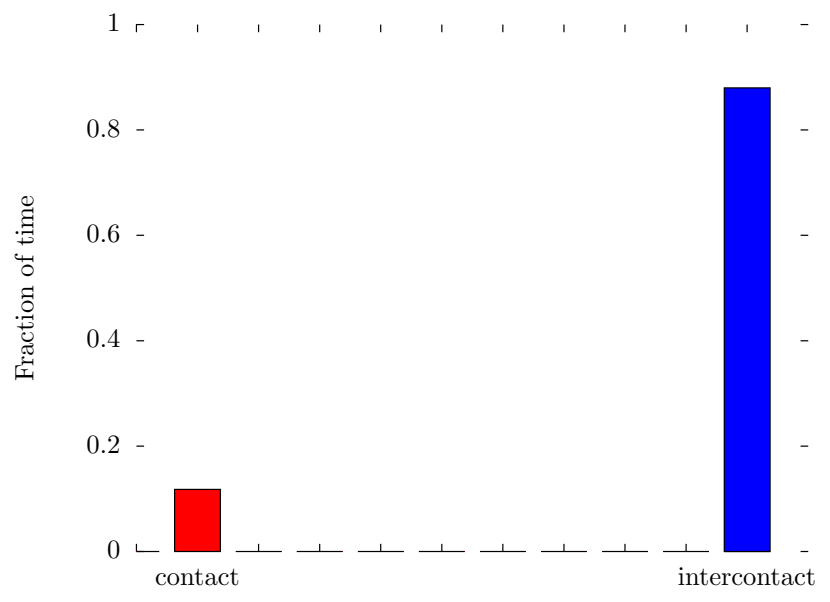

(a) Binary intercontact: nodes are in contact for $10 \%$ of the time and in traditional intercontact for $90 \%$ of the time.

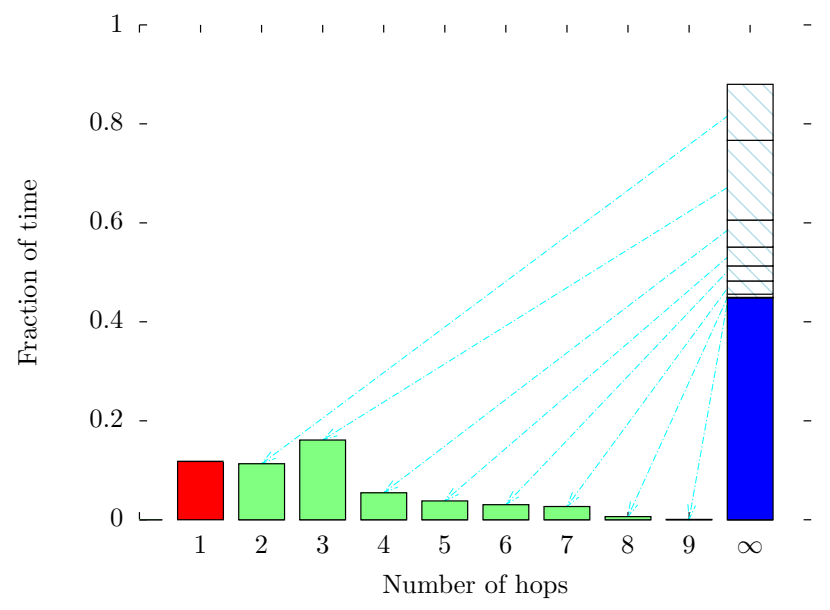

(b) $N$-ary vision. Like in Fig. 2(a), nodes spend $10 \%$ the time in contact but, in reality, they dwell at a distance 2 for around $10 \%$, at a distance 3 for $16 \%$. Real intercontact (i.e., pathless intercontact) deprived of multi-hop path represent only $50 \%$ of the timeline $(\infty)$.

Fig. 2. Example of time-distance distribution for a random trip-based dataset.

\section{B. A motivating example}

In Fig. 2, we generated synthetic traces using the Random Trip mobility model and compared conventional intercontact with our $n$-ary vision for one pair of nodes. When using the binary definition for intercontact (Fig. 2(a)), the two nodes under consideration spend $10 \%$ of their time in contact (i.e., 1 hop) and 90\% of their time in intercontact. In Fig. 2(b), we show the results using the $n$-ary definition. Observe that, even though while in intercontact, nodes stay at a 2-hop distance during $10 \%$ of the experiment duration, at a 3-hop distance for $16 \%$, and at a 4 -hop distance for 5\% (and also some little percentage at other distances). Pathless intercontacts account for less than $50 \%$ of the time (compared to the $90 \%$ observed by the traditional definition). In practice, protocol designers could benefit from favorable intercontacts as a mean to use multi-hop transfers (likely reducing delay) instead of playing only with the "wait" parameter.

The contact, favorable intercontact, and pathless intercon- 
tact triplet is the new ternary classification we suggest toward more detailed DTN characterization.

\section{Potential usages}

MANET, DTN, or hybrid solutions. With the impressive literature on ad hoc networks, rejecting the MANET-DTN correlation would be a waste. With a more accurate intercontact understanding, a node may decide to use MANET communications instead of a pure DTN algorithm or even discriminate between wireless interfaces like Wi-Fi, Bluetooth, $3 \mathrm{G}$ or LTE. Any protocol would be able to choose the relevant strategy depending on its current status. Ideally, we can think of new hybrid approaches using ad hoc and disruption-tolerant approaches together.

Interesting forwarding situations. For routing concerns, sensing a node's vicinity up to a 2 or 3 -hop distance sounds appealing. These situations embody end-to-end transmission opportunities via few close relays and therefore, low costs concerning delivery delays. We also observe higher distance paths. However we can question path stability and delays inferred by the store and wait process. Path of higher distances are less frequent than distance 2 or 3 paths. Moreover, a node can easily obtain his neighborhood up to a distance 2 or 3 running a simple link state protocol.

Oblivious communities detection. An important class of DTN movements are human-driven. Their topology relates to individuals with phenomenon like Milgram's Familiar Stranger [9]. Identifying prominent intercontact patterns could point out nodes with additional properties to leverage. We could also use our improved intercontact patterns to study induced communities evolution, their motion and stability. Intercontact patterns reflect more than a distance to another node, they disclose inherent sociostructure information and above all, new relevant DTN characteristics.

\section{DATASETS}

We observed in the previous section that the $n$-ary intercontact concept does provide further insights in the case of a synthetic trace. But what about real situations? To answer this question, we choose several well-known DTN datasets available to the research community.

Infocom05 is a dataset obtained in a conference scenario [10]. It involved 41 iMotes that captured contacts between participants during around 5 days of the conference. We focus on a 12-hour period of the second day, as it presents a higher network activity. In the Infocom05 experiment, each iMote performs a scan every 120 seconds.

Infocom06 is another conference-based dataset with 78 iMotes for 3 days [10]. We consider the second day to avoid the biases of the beginning and end of the experiment. Other parameters remain the same as in Infocom05.

Rollernet involved 62 iMotes during a 3-hour Rollerblade tour in Paris [11]. Leguay et al. set a shorter scanning granularity of 15 seconds. This dataset represent a highly dynamic situation with inherent strong social relationships.

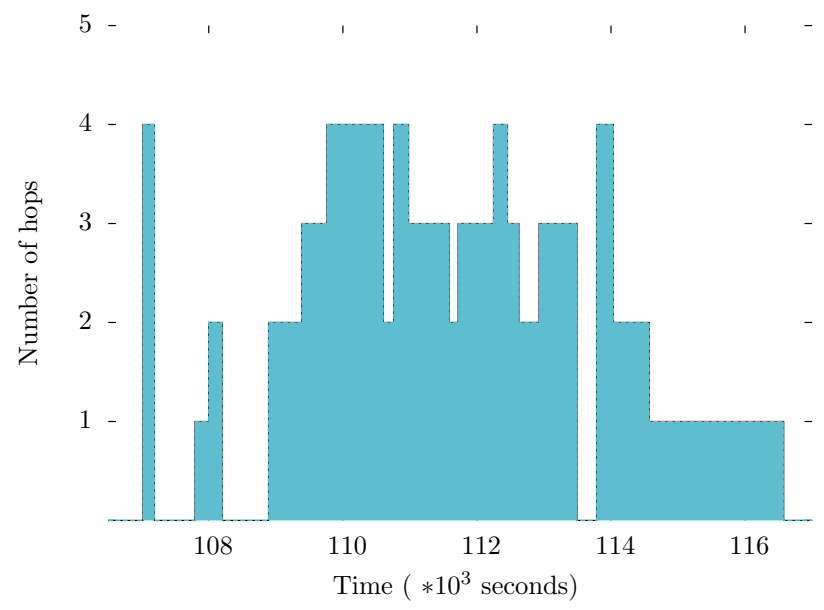

Fig. 3. In a timeline, we observe the mutual shortest path length between two given nodes in a network. At the beginning, they stay at a 4-hop distance for a few instants then have no more contemporaneous path between them. They are in pathless intercontact. At 108,000 seconds, they are back in contact and drift away to a distance 2 until there is no path. For the longer part of this timeline, nodes bear favorable intercontact properties.

KAIST and NewYork come from an experiment performed by NCSU researchers [12]. Unlike Infocom05 or Rollernet, they do not represent contact traces but GPS coordinates. We converted them via movement simulation and assigned every node with a 10-meter wireless range transmission (to emulate a Bluetooth situation, like in the datasets above). KAIST has 92 nodes for campus-based movements and NewYork has 39 nodes in a city wide measurement.

\section{RESULTS}

In this section, we evaluate our $n$-ary intercontact definition on the real-life DTN traces presented in the previous section. We first analyze the proportion of nodes bearing favorable intercontacts then observe the temporal characteristics of the parameters.

\section{A. Timelines}

We obtained timelines indicating the evolution of shortest paths between pairs of nodes in the network. Fig. 3 shows an example (a segment for the Infocom05 dataset). From 114,000 to 116,000 seconds, we can see nodes getting closer. Their shortest path length shrinking from 4-hop distance to 2-hop and finally dwelling at a 1-hop distance (in contact). We also observe the opposite phenomenon between 109,000 and 111,000 seconds, nodes are drifting away from distance 2 to distance 4 . For all pair of nodes, these timelines embed interesting behavioral patterns.

\section{B. Binary or $n$-ary intercontact?}

Based on our timelines, we represent the fraction of time two nodes stay in contact and the cumulated time they spend in favorable intercontact. We present these behaviors in Fig. 4. Each dot reflects the behavior of a pair of nodes. The closer the points are to the top left corner, the more they experience 


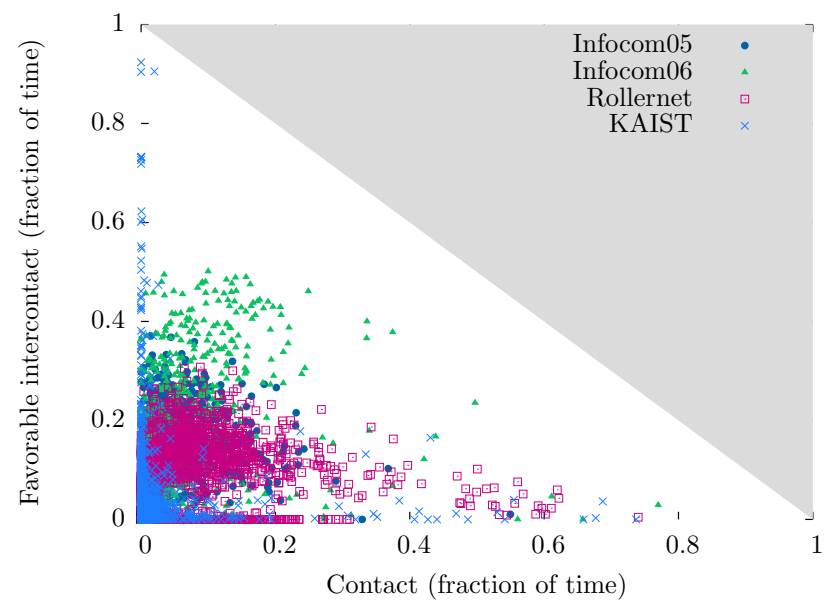

Fig. 4. Pairwise behavior according to the $n$-ary characterization. Each dot represents a pair of nodes. On the $x$-axis, we have the fraction of time they dwell in contact. On the $y$-axis, the fraction of time they observe favorable intercontacts. Note that, for the same density of contact, we can obtain a wide difference in favorable intercontact percentage.

favorable intercontacts and fewer direct contacts. This indicates that these nodes are frequently nearby (i.e., with endto-end paths to one another) but hardly experience a direct contact. Points strictly on the $x$-axis show pairs of nodes that are either in contact or in pure pathless intercontact, following the traditional DTN definition. Note that, as a significant share of the points fall in between, it means that, in practice, a non-negligible share of the pairs do show frequent favorable intercontacts.

We observe that a large portion of nodes display a significant fraction of time with end-to-end transmission capacities endorsed by contact and favorable intercontact. Nodes bearing these transmission capacities for up to $10 \%$ of their timeline (around 75 minutes except for Rollernet which is 6 minutes) represent: $70 \%$ of Infocom05 nodes, $29.7 \%$ of Infocom06, $5.8 \%$ of KAIST and $60.1 \%$ of Rollernet. In terms of duration, for their respective experiment span, more than $76 \%$ of Infocom05 nodes, 51\% of Infocom06, 19\% of KAIST and 83\% of Rollernet nodes have more than 20 minutes of end-to-end transmission possibilities.

The importance of favorable intercontacts in these datasets quantifies how inaccurate the binary intercontact characterization is. Its use ends up in massive transmission opportunities losses. In the next section, we notice how our ternary vision gathers oblivious sociostructure information.

\section{Sociostructure and favorable intercontact opportunities}

Pairs of nodes can be in contact or linked via one or more relay nodes on the shortest path between them. From Fig. 5 to Fig. 8, we present what we call an aggregated network sociostructure. There, we plotted (in layered mode) the number of connected pairs for each shortest distance. This means that layer 2 shows the amount of pairs connected via a 2-hop path, layer 3 via 3-hop paths, and so on. The bottom layer symbolizes the amount of pair of nodes in contact (i.e., 1 hop). For the sake of clarity, we also show

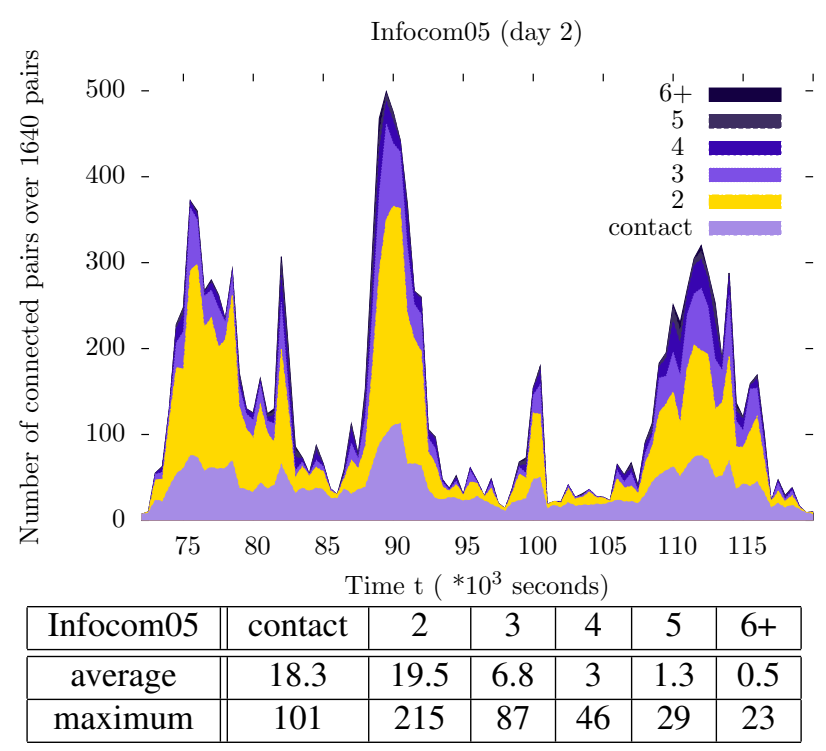

Fig. 5. In Infocom05, 2-hop transmission opportunities overcome direct contact ones. This phenomenon is emphasized in dense periods. In such dataset, end-to-end paths using one relay are omnipresent and should be leveraged on.

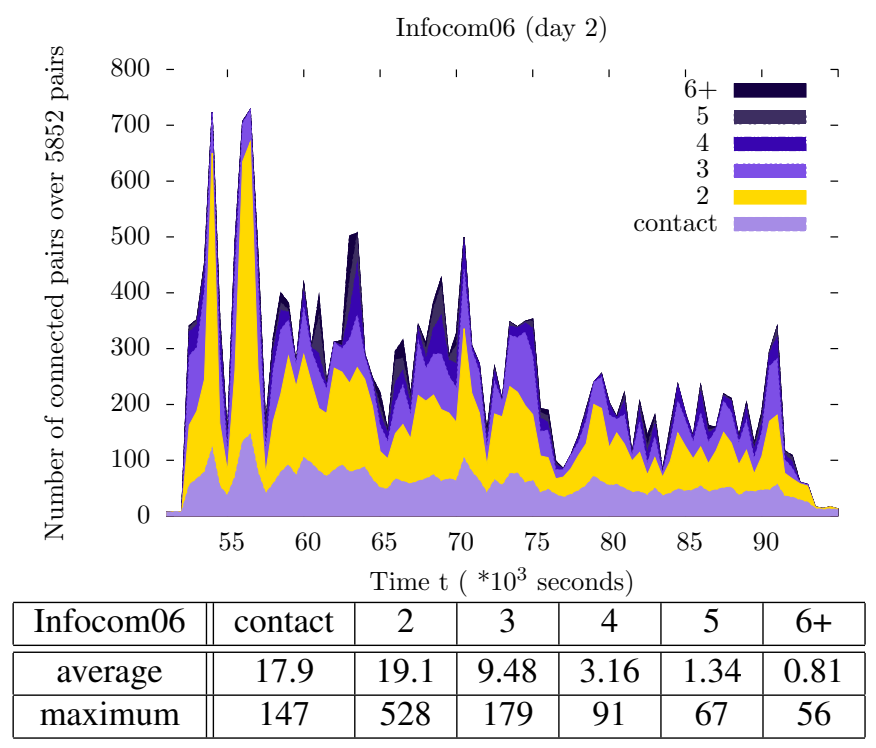

Fig. 6. Infocom06 verifies the same properties as Infocom05. 2-hop connectivity embodies more end-to-end transmission possibilities than direct contact.

representative values in corresponding tables underneath each plot. The first line indicates the number of pairs in average for each distance. We also display the maximum value for each distance. It is important to recall that such transmission opportunities are powerful as they only involve few relays that could reduce significantly end-to-end delays. Note for example the significant share observed for 2-hop paths.

In Fig. 5, we observe several peaks of connected pairs with their extended contact neighborhood. Given that Infocom05 is a conference-based measurement, we can correlate them with morning arrivals, lunch, afternoon break, and end of sessions. An unexpected visual observation is how 2-hop 


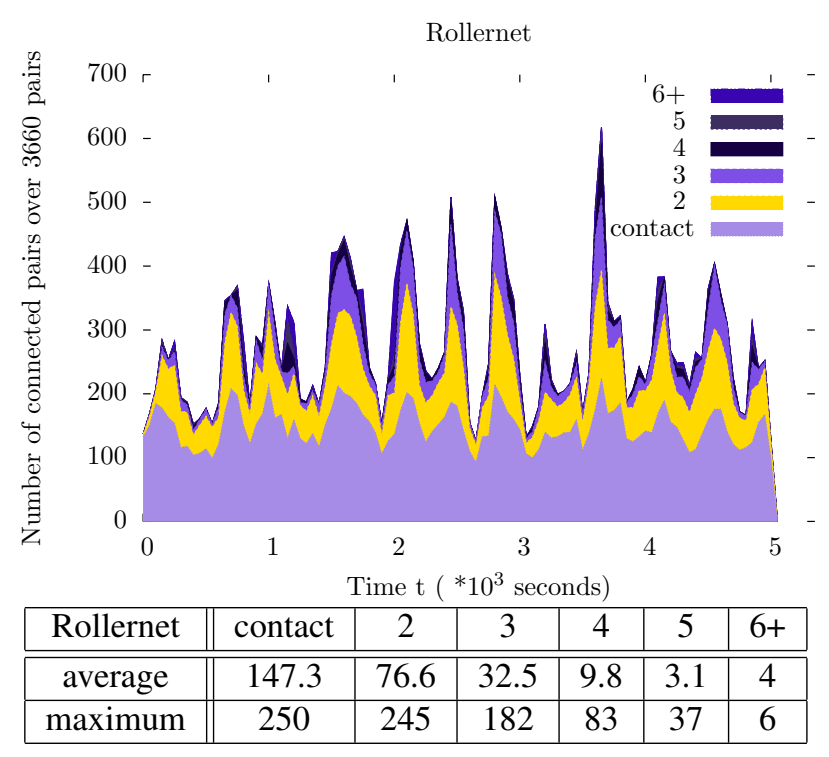

Fig. 7. Rollernet being a specific sport event with a tight population, most transmission opportunities come from direct contacts (1 hop). However, during the revolving phase of the accordion phenomenon (density peaks), we observe a growth in the number of 2+-hop paths.

favorable intercontacts overcome contact opportunities during these peaks of high density. These environments of highly connected crowds ignite favorable intercontacts. As a result, in such a scenario, favorable intercontact-based transmissions should be more helpful than direct contact transmissions or real DTN schemes.

For Infocom06, time divisions are less clear. However, we maintain our observation concerning 2-hop distance connected pairs (see Fig. 6). As a rule, 2+-hop favorable intercontact overthrows contact possibilities. Infocom06 is a quite dense dataset with a large amount of nodes at regular pedestrian speeds in a smaller surface than for other datasets we considered. This suggests that such environments, similar to many urban settings, are prone to have impressive extended end-toend communication possibilities.

In Fig. 7, we observe Rollernet's inherent accordion phenomenon, i.e., the sequential stretching and shrinking of the crowd due to urban obstacles preventing the crowd from moving forward [11]. Rollernet has a dynamic setting with a compulsory path. Nodes do not have as much movement liberty as they have in other datasets. Contacts are prominent in Rollernet, but still favorable intercontacts are noticeable in peaks of density.

Considering KAIST, contact opportunities may seem more frequent than favorable intercontacts. But, unlike previous datasets where connectivity quickly decreases with distance on average, we discover comparable connectivity between nodes for distance 2 to 5 . KAIST environment being a campus-based measurement, it has a sparser density with a lot of movement freedom. Students tend to stay where other students are, like in restaurants, buildings, libraries but they may not share stronger relationships than just being student in the same university so they do not form close groups. This is a possible explanation of how extended favorable intercontact state lingers here.

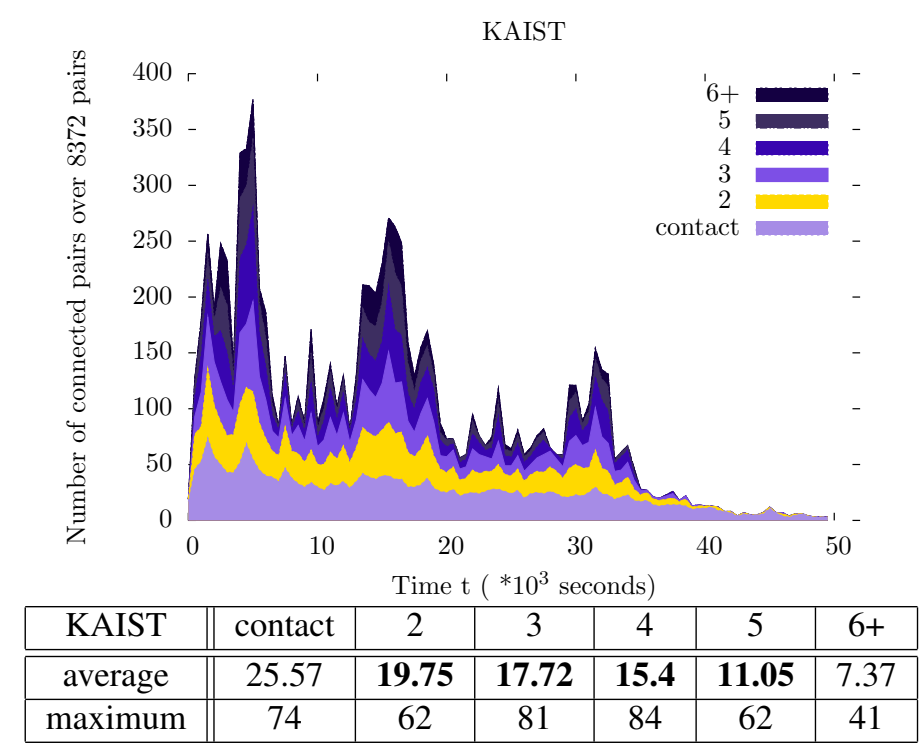

Fig. 8. KAIST is a campus-wide experiment. Students are likely to spread around alleys and buildings leading to dense areas at some points in time. Therefore, we have similar favorable intercontact proportion from distance 2 to 5. Opportunistic strategies clearly have room to spread in such a setting.

In any case, peaks indicate denser areas with more communications possibilities. We visually notice how contact opportunities represent only a minor part of end-to-end transmission opportunities.

\section{Pathless intercontact time characterization}

Until now, we have focused on the detection of favorable intercontacts, which are interesting for their native communication opportunities. However, understanding pathless intercontacts also bring interesting network indications. We will next focus on this aspect.

Chaintreau et al. performed intercontact time characterization for various existing datasets [13]. They used the traditional binary contact vision where nodes not in contact are in intercontact. We perform the same analysis but with pathless intercontact only.

In Fig. 9, we plot the CCDF of intercontact times, i.e., the probability for an intercontact to last for at least $t$ seconds. Dotted lines represent the distribution for binary intercontact vision whereas solid ones correspond to pathless intercontact. The overall aspect of the distributions remains the same with a general deviation to the left for the pathless intercontact CCDFs. This phenomenon is logical as we extended the contact notion, i.e., it reduces pathless intercontact durations when compared to initial binary intercontact. The contact extension only occurs in periods where the network is dense. As a result, pathless intercontact distribution is not an exact translation of binary intercontact one but keeps an overall similarity. This also means that our definition does not change the intercontact properties our community used to have when studying DTN patterns. So, we can still leverage on the existing forwarding strategies background like techniques based on power law opportunities with adjusted parameters [13]. In other words, our definition does not discard all the existing 

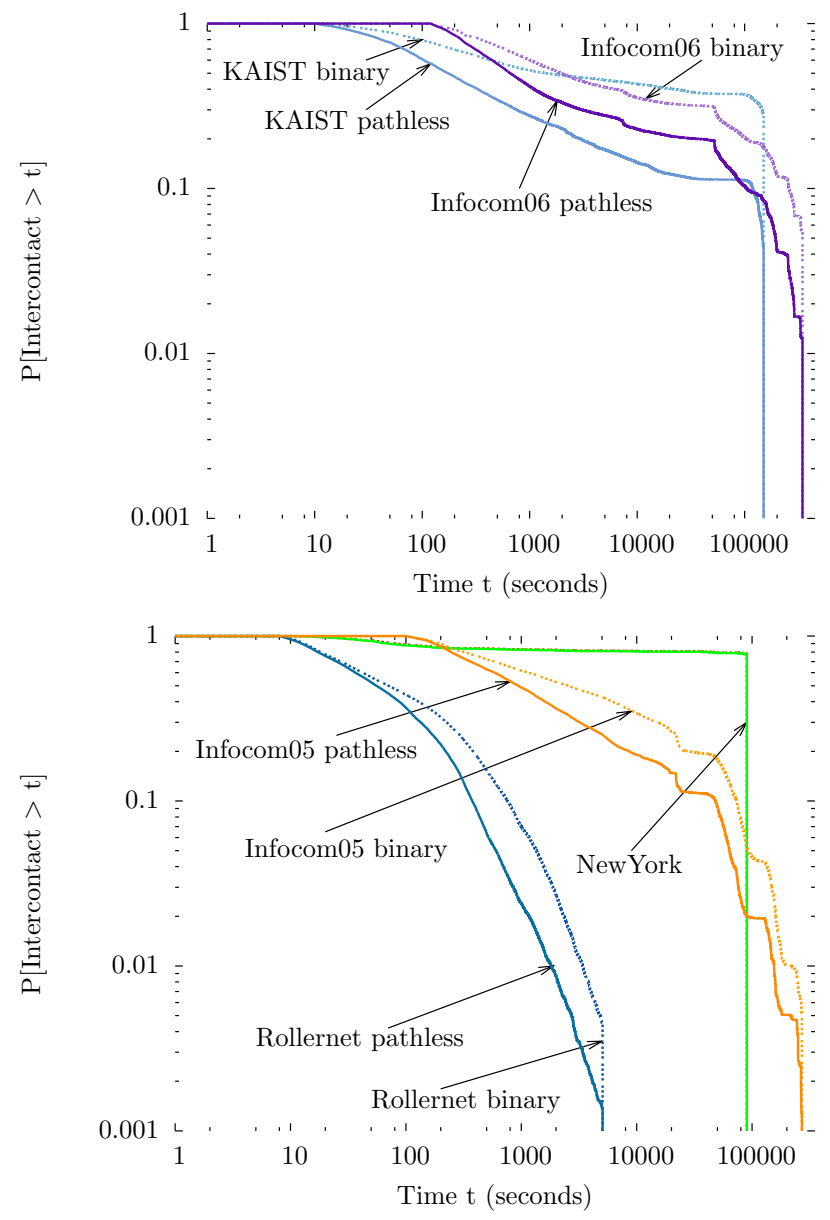

Fig. 9. Aggregated distribution of binary and pathless intercontact times. Pathless intercontact keeps the same overall distribution as binary intercontact with a small deviation on the bottom left. In the NewYork dataset, both distributions line up as we have almost no favorable intercontact. (log-scale on both axes.)

literature on DTN schemes; instead, it enhances it by providing new beneficial network information via extended contacts.

\section{SUMmARY AND OUTLOOK}

In this paper, we questioned the completeness of the binary intercontact vision where nodes are either in contact or in mere intercontact. We advocate for a new ternary vision in DTN characterization via a fine-grained intercontact definition. Our contribution, the $n$-ary intercontact, proposes to enhance the intercontact notion. We expose two intuitive concepts: favorable and pathless intercontacts. On a node-centered vision, favorable intercontacts bring new communication opportunities with the awareness of end-to-end paths. Whereas, pathless intercontacts include the previous binary intercontact abstraction and preserves any existing approaches relying on their distribution.

Our $n$-ary intercontact characterization is very compliant to DTN applications. It grasps what binary intercontact vision misses: the underlying MANET nature in DTN. It explicits new communication opportunities. Here, we intentionally introduced a simple definition for our community to integrate it in their every day approaches. We have shown for example that in Rollernet, Infocom05 and Infocom06 more than 50\% of the pairs of nodes display favorable intercontact opportunities.

We hope this work will bring incentives for our community to develop new communication solutions for DTNs. We think the binary intercontact hypothesis prevents disruption-tolerant approaches from achieving their optimal efficiency when more information about the network can only help. In a near future, we plan to propose hybrid protocols considering MANET and DTN transmission opportunities alongside to leverage communication opportunities revealed by the $n$-ary intercontact characterization.

\section{ACKNOWLEDGMENT}

This work is partially supported by the ANR project CROWD under contract ANR- 08-VERS-006. The authors would like to thank John Whitbeck for its help preparing the manuscript.

\section{REFERENCES}

[1] K. Fall, "A delay-tolerant network architecture for challenged internets," in ACM SIGCOMM, 2003.

[2] P. Hui, J. Crowcroft, and E. Yoneki, "Bubble rap: social-based forwarding in delay tolerant networks," in ACM Mobihoc, New York, NY, USA, 2008.

[3] A. Lindgren, A. Doria, and O. Schelén, "Probabilistic routing in intermittently connected networks," SIGMOBILE Mob. Comput. Commun. Rev., vol. 7, July 2003.

[4] T. Spyropoulos, K. Psounis, and C. S. Raghavendra, "Spray and wait: an efficient routing scheme for intermittently connected mobile networks," in ACM SIGCOMM workshop on Delay-tolerant networking, 2005.

[5] J. Whitbeck and V. Conan, "Hymad: Hybrid dtn-manet routing for dense and highly dynamic wireless networks," Computer Communications, vol. 33, August 2010.

[6] M. Musolesi and C. Mascolo, "Mobility Models for Systems Evaluation. A Survey," in Middleware for Network Eccentric and Mobile Applications, February 2009, pp. 43-62.

[7] S. Gaito, E. Pagani, and G. P. Rossi, "Opportunistic forwarding in workplaces," in ACM SIGCOMM workshop on Online Social Networks, 2009.

[8] V. Borrel, M. H. Ammar, and E. W. Zegura, "Understanding the wireless and mobile network space: a routing-centered classification," in $A C M$ MobiCom workshop on Challenged Networks.

[9] E. Goodman and E. Paulos, "The familiar stranger: anxiety, comfort, and play in public places," in ACM SIGCHI, 2004.

[10] A. Chaintreau, P. Hui, J. Crowcroft, C. Diot, R. Gass, and J. Scott, "Impact of human mobility on opportunistic forwarding algorithms," TMC, vol. 6, no. 6, pp. 606-620, 2007.

[11] P. U. Tournoux, J. Leguay, F. Benbadis, V. Conan, M. Dias de Amorim, and J. Whitbeck, "The accordion phenomenon: Analysis, characterization, and impact on dtn routing," in IEEE Infocom.

[12] I. Rhee, M. Shin, S. Hong, K. Lee, and S. Chong, "On the levy-walk nature of human mobility." in IEEE INFOCOM, 2008.

[13] A. Chaintreau, A. Mtibaa, L. Massoulie, and C. Diot, "The diameter of opportunistic mobile networks," in ACM Conext, 2007. 